\title{
Visualizing Activity on Wikipedia with Chromograms
}

\author{
Martin Wattenberg ${ }^{1}$, Fernanda B. Viégas ${ }^{1}$, and Katherine Hollenbach ${ }^{2}$ \\ ${ }^{1}$ IBM Research \\ ${ }^{2}$ Massachusetts Institute of Technology \\ \{mwatten, viegasf\}@us.ibm.com, kjhollen@mit.edu
}

\begin{abstract}
To investigate how participants in peer production systems allocate their time, we examine editing activity on Wikipedia, the well-known online encyclopedia. To analyze the huge edit histories of the site's administrators we introduce a visualization technique, the chromogram, that can display very long textual sequences through a simple color coding scheme. Using chromograms we describe a set of characteristic editing patterns. In addition to confirming known patterns, such reacting to vandalism events, we identify a distinct class of organized systematic activities. We discuss how both reactive and systematic strategies shed light on self-allocation of effort in Wikipedia, and how they may pertain to other peer-production systems.
\end{abstract}

Keywords: Wikipedia, Visualization, Peer Production, Visualization.

\section{Introduction}

The past decade has seen a surge of activity in peer-production projects, where work is conducted by volunteers who make their own decisions about what to work on and when to work on it. Benkler [2] has argued that the success of these projects is partly due to the efficiency with which individuals allocate their own efforts. While several studies have examined what motivates contributors to these projects and the processes behind them $[9,11]$, the mechanisms by which individuals allocate their effort are not fully understood.

One of the archetypes of peer production is Wikipedia, an online encyclopedia which anyone on the internet may edit. Not only is Wikipedia an influential example of peer production, but it is one for which a great deal of information is available. The Wikipedia community makes public nearly complete data on article edits and the discussions surrounding them.

Our investigation is based on this data, in particular the edit histories of Wikipedia "administrators," a key subset of active contributors. Since these histories are large and unstructured the analysis is a challenging problem. Our solution is to use a new visualization method, which we call a chromogram, that converts textual data to colors, producing a data-dense display that can fit a vast edit history onto a single screen. Because the method is simple and general we believe it is of interest in its own right, with potential applications in other areas.

We apply the chromogram technique to the activities of Wikipedia administrators. One clear result is heterogeneity of individuals' work habits at every time scale. 
Almost every editor we studied engaged in task switching, rarely working on one article or type of task continuously. At the same time, most editors we studied also had at least one area of concentration: this focus could relate to content categories (for example ships, television shows, or alcoholic beverages) or to process (e.g., fighting vandalism, welcoming new users).

We identify two broad classes of focal activities: reactive edits (such as repairing vandalism), and "systematic" projects characterized by a sequence of repetitive activity. A common systematic strategy, for instance, is to make the same type of edit to a long sequence of related pages in alphabetical order by title.

We conclude by discussing potential explanations for these phenomena. In particular, we suggest that both the reactive and systematic strategies arise in response to the fact that work in Wikipedia often consists of a large number of very small tasks. It has been hypothesized [2] that a division of labor into many small modular tasks is a general characteristic of successful peer production projects, and so we discuss how our findings may relate to other peer production systems. We also show how these usage patterns suggest design principles for systems aimed at collaborative creation of content.

\section{Background and Related Work}

It is worth reviewing some of the mechanics of Wikipedia and how they may affect decisions about work. We start by narrowing our view to the English language version. This site is made up of several different types of pages lying within distinct "namespaces". The "Main" namespace holds the actual encyclopedia articles. Other key areas include "Main:Talk" where discussions about particular articles take place, "Image" where pictures are kept, and "Wikipedia," where guidelines are held. See [Viegas07] for more details.

Wikipedia's prominence, influence and transparency make it a natural target for research. A recent set of studies have addressed social and technical aspects of the system. Viégas et al. [16, 17] have investigated the collaboration and selforganization surrounding articles. Forte \& Bruckman [6] have examined the incentives for individuals to contribute to Wikipedia, and Bryant et al. [4] suggested that people can make the transition from newcomer to Wikipedia expert via legitimate peripheral participation [10].

Many of the questions asked about Wikipedia-how does a system convince people to contribute their time for free? Why do people do it?-have also been investigated in the realm of open-source development. Several studies [9, 11], have examined what motivates people to perform for free what is normally highly paid work. A common thread in the findings is that contributors view open-source projects as an opportunity for intellectual stimulation and a chance to make tools for their own use.

Yochai Benkler [2] posits that Wikipedia and open-source development both fall into a general category of "peer production" systems. His argument is grounded in a broad review of existing practices and suggests that peer production systems thrive when they break work into fine-grained, modular tasks. Benkler argues that these systems can work well partly because the volunteer participants can allocate their own labor very efficiently. 
The mechanics of this efficient allocation, however, remain mysterious. Indeed, none of the treatments described above examines the details of the day-to-day choices of individual participants. The closest may be the work by Bryant et al. [4]; our analysis of detailed edit histories is complementary to theirs, which is based on in-depth interviews with Wikipedia members. Just as with recent investigations of open-source projects and work on online communities such as listservs [5] the methods to date have largely relied on interviews and surveys of community members.

Before describing our own methods and results, it is worth discussing some of the ways that Wikipedia editors might choose what to work on. First, users often look for articles on specific topics of interest [4]. They may also respond to requests for help on a Talk page [17]. Technical mechanisms exist as well. Several investigations have noted the importance of watchlists, which allow users to monitor all changes to a customized set of pages, along with the "recent changes" and "random article" pages [6, 16, 17].

A final explicit way that Wikipedians organize work is through "Wikiprojects". A wikiproject is a set of pages that defines a focused area of activity, describing the scope of work, listing participants and work items, and offering identifying markers that can be placed on talk pages to advertise the project. Wikiprojects have distinctive social atmospheres. On "Wikiproject: Mathematics" a typical participant introduces himself with, "Ph.D. in mathematics from Caltech, with a specialization in mathematical logic" while on "Wikiproject: Beer" a representative self-description reads only "mmm, beer." Each project thus represents a community of interest.

\section{Methods}

The raw material for our investigation is the edit histories of Wikipedia administrators. An administrator (or "admin") is a user who has access to special functions such as page deletion and protection. A full description of the role of admins in the community and how they are selected is beyond the scope of this paper, but as a trusted, active, and influential set of users they play a core role in Wikipedia. In some respects they are analogous to "committers" on an open-source software project. Furthermore, such users are one of the big mysteries of Wikipedia: some average more than 100 edits per day over the course of a year, which seems surprising for volunteer labor. As of 2006, roughly $14 \%$ of edits on the English Wikipedia site have come from administrators. ${ }^{1}$

\subsection{Data Collection}

To ensure a sample of users with a substantial history as admins, we selected all "active" administrators as of October 2005. For these users we downloaded edit histories that ran until August 2006. The list contained histories for 514 users, but irregularities in the data format meant five of these had to be discarded, leaving a total of 509 histories.

An edit history for an individual user is a sequence of timestamped edit events, where an event consists of the title of the article edited and an optional user comment.

\footnotetext{
${ }^{1}$ Based on a 2006 Fig. of $81,132,479$ edits from [23] and a total of 10,972,403 edits from more than 900 admins reported on [22].
} 
We observe that Wikipedia comments tend to follow certain conventions. They are usually brief, to the point, and employ standard terms for user actions. For example, "rv commercial promotion" describes the action of reverting an edit that had been made to promote a company.

\subsection{Privacy}

The data discussed in this paper is publicly available on the Wikipedia website, both as an easy-to-download database dump and in aggregated form via prominently linked HTML pages. In addition editors have agreed that their work will be released under the GFDL license [7], which grants a wide range of permissions to third parties to copy the data. Nonetheless, individuals may not expect their data to be analyzed as we have done in this paper, so we anonymized user names in all visualization diagrams and discussions. We have also made efforts to omit ancillary information that might easily identify the real usernames.

\subsection{Basic Descriptive Statistics}

Some basic statistics provide a sense of the data. Of the 509 selected administrators, the number of edits per person ranged from 789 to 122,387. The average number of edits was 16,704 and the median was 12,337. At the high end, this works out to an edit roughly every 10 minutes since May 2004! Admins spread their work over many pages, touching an average of 7,872 different titles, ranging from a minimum of 322 to a maximum of 87,976 .

\subsection{Exploratory Analysis}

How might one analyze these histories, going beyond simple descriptive statistics? To begin with, consider an abstracted version of the problem: how to study a sequence of timestamped text "tokens" (representing either comments or article titles). The distinguishing features of the data we face are:

1. Large scale: on the order of 100,000 events.

2. Diversity: up to 80,000 distinct text tokens.

3. Irregular structure: most edit histories we looked at had few precisely repeated subsequences or even long runs of a single token.

These three qualities make analyzing the data a challenge. The large scale means simply reading lists of edits is overwhelming. The fact that the data is textual rather than numeric rules out a range of statistical methods aimed at time series.

\section{The Chromogram Visualization Technique}

For these reasons we decided to use a visual exploratory method. While there is significant work in visualizing sequences of non-numerical data, the large space of tokens and irregularity again cause difficulty in applying existing techniques. Methods such as arc diagrams [20] that rely on finding precisely repeated subsequences do not work. 
Techniques that do handle large, noisy sequences of discrete events, such as those used in visualizing traces from software profilers (e.g. [3]) or development activity [13]), frequently rely on color-coding. The idea is to avoid the use of lengthy labels by representing tokens by colors; this tactic allows extremely efficient use of space.

Typically color coding either relates to a small set of discrete values for which distinctive colors can be chosen by hand, or take continuous values which can be naturally mapped to a portion of color space. In our case, however, mapping text tokens to colors is a subtler problem. For any mapping of thousands of different strings to colors, many strings will inevitably receive nearly indistinguishable colors. To minimize this problem it is obviously desirable to make use of a large color space. In addition, it is desirable that the color mapping be consistent from one data set to the next, so users might recognize the colors of common words. Finally, one might hope that "similar" words would receive similar colors.

To balance these criteria, we decided on a scheme in which the first three letters of a string determine the color of its representation. The first letter determines the hue; the second letter the saturation, and the third the brightness. Saturation and brightness were both kept in a restricted range so that the hue was easily perceived. (Many variations of this method were tried, but this seemed to bring out patterns in the data better than other hue/saturation/brightness permutations, possibly due to the categorical nature of hue perception. We also tried mapping the first three letter values to the three RGB components but the resulting diagrams were hard to read.) As a special case, titles or comments that begin with a number were converted to a shade of gray based on the initial digits.

Fig. 1 shows how sample text tokens - in this case, typical words from user comments in Wikipedia-translate into colors. Note, for example, how "added" and "arbitration" differ largely in saturation, while "articles" and "arbitration" differ only in brightness.

At first this may look like an arbitrary way to encode text. Strings with opposite meanings can have identical colors (terrible and terrific) and strings with similar meanings can map to contrasting colors ( $d o g$ vs. the $d o g$ ). Similar colors may be hard to discriminate, and the perception of a given color will be influenced by its neighbors. The encoding does meet our criteria, however, of spreading colors over a large section of color space and of remaining consistent from one sequence to the next. In practice, moreover, we found the method effective at revealing structural features. The Findings section illustrates the broad variety of patterns that this encoding exposes, and in the Discussion section we suggest reasons for this unexpected efficacy.

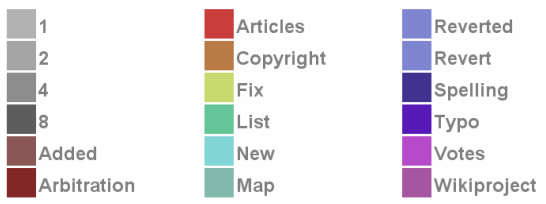

Fig. 1. Sample Translations from Words to Colors 


\subsection{Drawing the Sequence}

The color mapping described above allows us to display long edit histories on a single screen, using what we call a "chromogram". The idea of a chromogram, as illustrated in Fig. 2, is straightforward. Fig. 2a shows a hypothetical list of edits. To visualize this sequence, focusing on the comments, we can create a histogram (2b) where each row corresponds to a day, and contains one rectangle for each edit, ordered by time and colored with the alphabetical scheme above. Fig. 2c shows a "block view," in which edits are simply placed in a block, time-ordered left to right and then top to bottom (just as we read). This saves space at the expense of obscuring temporal rhythms; to keep some idea of time we label the beginning of each row with the date of the first edit in the row. Finally, Fig. 2d shows a compressed block view: here the rectangles for each edit are made small and labels are omitted.

The distinguishing feature of a chromogram is the method of mapping text to color. The layout is simple; similar methods have been used in systems such as the pixeloriented visualizations of Keim [8]. The geometry is also similar to actograms, e.g., as used by Begole et al. [1] to visualize work rhythms. Unlike an actogram, however, the $x$-axis encodes not time but sequence, which allows more efficient use of space for bursty sets of activities.

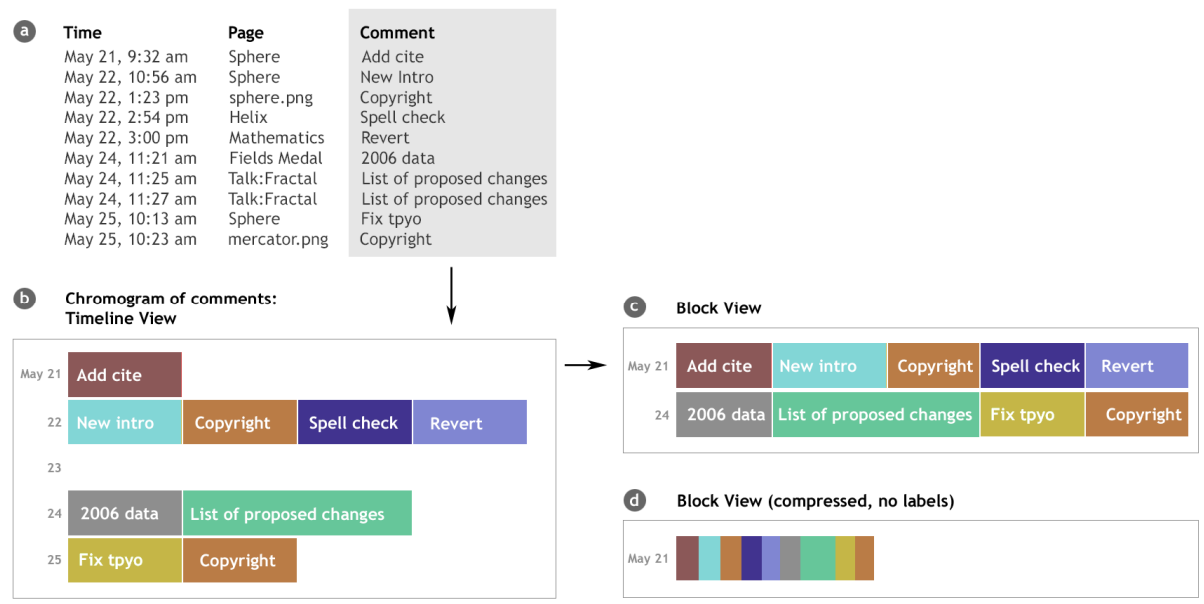

Fig. 2. Creating a Chromogram

\subsection{The Chromogram Application}

We implemented this visualization technique in a Java desktop application. The software reads edit history files in a tabular format and creates a chromogram of the results. Fig. 3 shows a screenshot, with blurring where text might identify the editor. Here the chromogram shows a block view (as in Fig. 2c).

The labels on the $y$-axis show the date of the first edit in each of the rows and the labels on the $x$-axis count the numbers of edits in each row's sequence. As the user 
moves the mouse over edit rectangles in the chromogram, a popup window shows details about article title, comment, and date. In addition to the central chromogram, some other user interface elements proved helpful. The top gray bar holds elements that let an analyst switch between visualizations of edit comments and titles of edited articles, and search for edits that contain particular words or phrases.

At right is a list of the most common words in the chromogram, arranged alphabetically. This list provides a quick color key for the most frequent titles or comments. Next to each word on the list is a bar indicating its frequency, which makes it easy for a user to get a sense of where and how the editor is spending their time. The items on the list are clickable so that a user can highlight only the edits that begin with a particular word of interest.

Fig. 4 shows a timeline chromogram view (corresponding to Fig. 2b). The data is the same as in Fig. 3. Because the timeline view is usually far less space-efficient than the block view, a scrollbar is often needed. The vertical gray stripe in Fig. 4 shows the scrollbar, which contains a histogram showing a wider view of the distribution of edits. The orange area highlights the region currently on screen. Note that Fig. 3, the block view, displays the entire sequence of edits on screen.

\section{Findings: Patterns of Activity}

Using the chromogram application we examined chromograms for all 509 admins. For each chromogram we looked at both the comment view and title view. This study suggested a number of patterns, which we describe below. For quantitative patterns we were able to corroborate our findings by computing statistics on the total set of administrators.

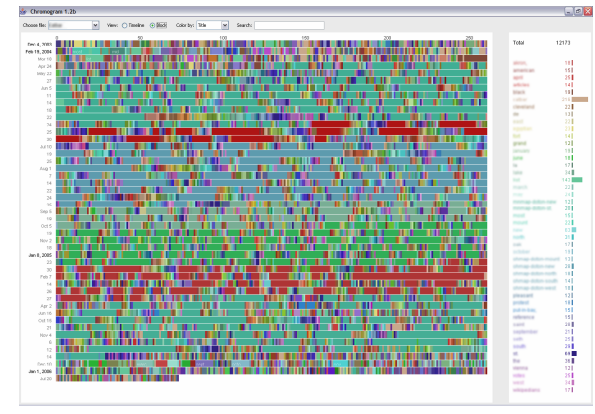

Fig. 3. The Chromogram Application: Block Fig. 4. The Chromogram Application: TimeView

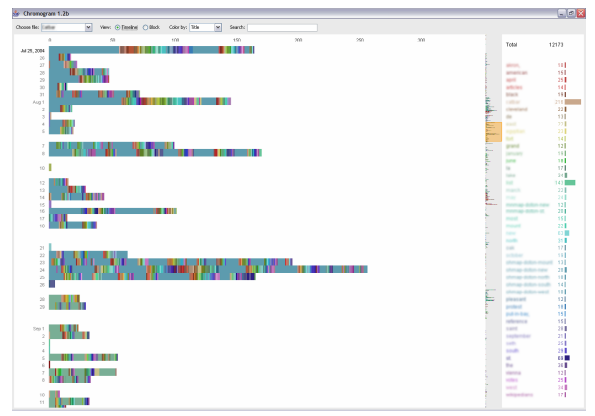

line View, same data as Fig. 3

\subsection{The Shape of Activity: Focal Tasks + Sporadic Edits}

Although each editor we examined had a distinctive activity chromogram, some commonalities emerged. First, activity often occurred in bursts. The temporal view made this clear, as in Fig. 5. (To save space, in this and several subsequent Figures we show only a subset of the full chromogram.) This comment chromogram shows 
activity over the course of nine days. The first burst is largely typo fixing and the second burst-peaking at more than 300 edits in a day-involves adding disambiguation messages to pages. These bursts of activity correlate with type of edit, but we also saw patterns related to article content and calendar features such as weekends, weekdays, and holidays.

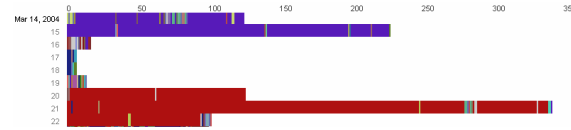

Fig. 5. Timeline comment chromogram with activity bursts. Blue: typo removal. Red: adding disambiguation messages.

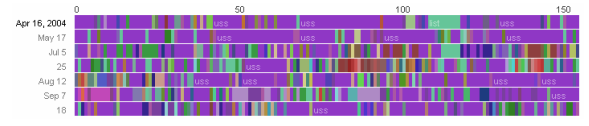

Fig. 6. Title chromogram: purple edits relate to U.S. ships, green to lists of ships

A second feature seen in the chromograms was switching between articles and between tasks. Editors seemed to move between several activities, sometimes at a small time scale and sometimes at a large one. For $99 \%$ of admins studied, the majority of edits were followed by an edit to a different article. Sequences of consecutive edits to a single article were generally short in comparison to the lengths of edit histories overall. Across all admins, the average ratio of a user's longest sequence of singlearticle edits to the total number of edits was below 0.01 .

Despite the heterogeneous nature of the activity, a degree of organization was evident in many user histories. In particular, many editors had a small set of tasks, defined by a set of similar edit comments (e.g., "revert vandalism") or related article titles (e.g., wine-related topics), that comprised a significant fraction of their edits. We term such an activity a focal task. Users displayed a great diversity of focal tasks, but they seemed to fall into two main categories: systematic activites and reactive actions. We describe both below.

\subsection{Systematic Activities}

Many users engaged in what we called systematic activities: that is, a sustained related sequence of edits. Some editors seemed to concentrate on particular topic areas. Fig. 6, a title chromogram, is typical: this user focused on naval history. The dominant color is a purple shade that corresponds to the prefix "USS" (United States Ship) used in the names of American naval vessels. Occasional edits with a different color are evident, but exploration of the data shows that they remain on the naval theme. Many of the green edits are to lists of ships, e.g., "List of all ships in the United States Navy, M" and "List of U.S. Military Vessels Named After Women."

Despite the intense topic focus the actual types of edits are diverse, as the comment chromogram for the same user and time period shows (Fig. 7). Although certain shades are frequently seen (the light brown corresponds to adding category information), the many different colors reflect many different actions: adding images, changing content, and so on. 


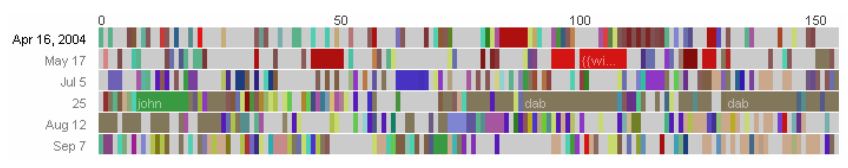

Fig. 7. Comment chromogram for same edits as Fig. 6: Highly irregular

Fig. 8 shows a title-based chromogram of a different type of systematic activity, covering roughly 900 edits over the course of four days in 2005. This user is "stub sorting," or putting category information in the small incomplete articles known as "stubs." The most visually interesting feature of this chromogram is the rough rainbow pattern: there is a clearly visible progression through the hues of the spectrum. Since the hue of an item represents the position of the first letter in the alphabet, this user seems to be methodically moving through an alphabetically ordered list of tasks.

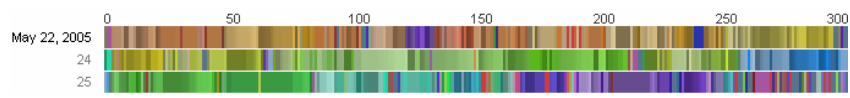

Fig. 8. Rainbows: Alphabetical order effects in a title chromogram for 900 edits

The actual subjects of the articles are diverse, ranging from zoology ("Humphead wrasse") to pop culture: "Push (Professional Wrestling)". How did this editor find this sequence? The comments for the edits tell the answer: each edit refers to the stub-sorting wikiproject, where the admin is a listed participant and where lists are kept of stubs to be sorted.

We also observed list-guided activities that did not seem to be driven by Wikiprojects, but rather by lists in the main content area of the encyclopedia. One example can be seen in Fig. 9. Recall that gray items in our chromogram represent numbers. This sequence shows an editor making a systematic, ordered series of changes to a set of pages representing yearly compilations (e.g., "1922 in Literature"). The ordering is made visible through the light-dark gradient of some of the sequences in the middle row, indicating an ordered set of editing of pages starting with 1 to pages starting with 9. There is no Wikiproject on years in literature, but there is a "List of Years In Literature" article that links to pages edited by this user.

It is also worth noting that in this history a diverse set of edits interrupted the systematic edits. Interspersed with the gray areas are bars of many different colors. These represent changes to other content areas, for instance reverting an edit on the "Torah" article; correcting spelling on a television show page; editing articles on famous mathematical conjectures.

The pattern of alphanumerically ordered edits turned out be common. We found extended, strictly increasing alphabetical sequences of titles in many revision histories.

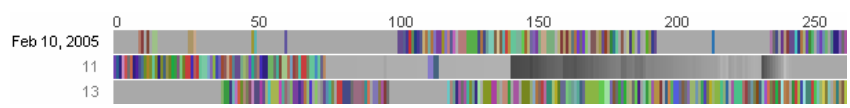

Fig. 9. Title chromogram: edits on pages with numerical titles (in gray) 
(We say "strict" to exclude sequences that contain the same title twice in a row, so that a long sequence of edits to the same article does not count.) Although one would expect to see some short ordered sequences by chance, what we observed went well beyond random effects. A simple test illustrates this point. If $p(n, k)$ represents the probability of a strictly increasing alphabetical subsequence of length $k$ occurring in a sequence of $n$ revisions, a straightforward calculation shows

$$
p(n, k)<(n-k+1) / k ! \text {. }
$$

By this measure, 307 out of 509 (60\%) of admin revision histories contained an alphabetically ordered subsequence of titles whose length was statistically significant. More precisely, 307 histories had a length $n$ and an alphabetical subsequence of length $k$ with $p(n, k)<10^{-5}<.01 / 509$. In 13 histories we observed alphabetical runs of more than 100 consecutive titles.

A natural question is whether ordering effects can be seen in Wikipedia itself. One may conjecture that if alphabetical lists guide a portion of work then titles that appear early in the alphabet may see the most edits. In fact analysis of a 2005 snapshot of Wikipedia data does suggest a weak negative relationship between alphabetical order and editing activity. The Spearman rank correlation between alphabetical order of first letter of article title and average number of edits is $-0.47(p<.05)$. A full investigation of this phenomenon is beyond the scope of this paper, but it would be interesting to test this trend by performing the same analysis on foreign-language versions of Wikipedia.

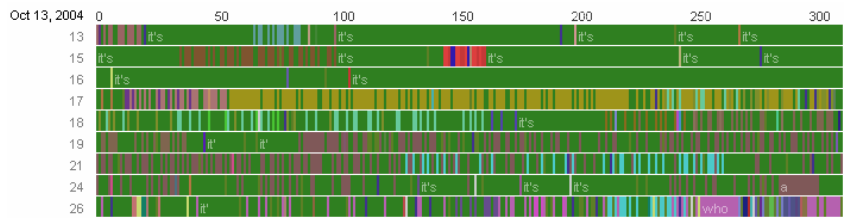

Fig. 10. Comment chromogram of about 2,900 copy edits. Green items are changes from "it's" to "its".

Stub-sorting is just one example of a whole range of organizational activities. Other organizational tasks include the creation of disambiguation pages and redirection pages. Yet another type of project involves "cleaning" a set of pages with known problems. Cleaning can cover grammar, spelling, or wiki syntax. It may also involve adding or modifying templates, changing formatting, or searching for copyright violations. An example is shown in the comment chromogram of Fig. 10.

A second common type of systematic activity was editing associated with maps and images. Fig. 11 shows a pattern we observed in several admins: switching between uploading images and then editing articles to include them. In the case of Fig. 11, which encompasses about 1,500 edits made over the course of a single week, the interspersed rainbow-like segments represent article edits, and the patches of near-uniform color represent images. The uniformity of color stems from naming conventions for these images: at top are maps of regions in Maryland, and their names all begin with "MDMap". 


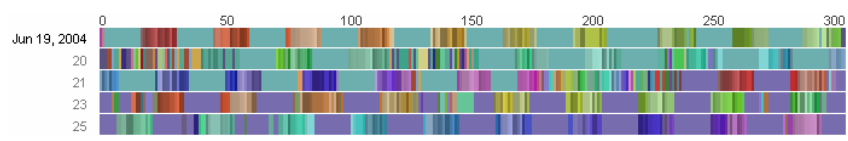

Fig. 11. Title chromogram: Switching between images (purple and light blue) and articles (rainbows)

A final type of systematic activity involves the use of "bots," or programs for making automated edits. Certain admins have written these programs as labor-saving devices. Because bots are technically users in Wikipedia, we could examine their edits histories in the same way we examined the activities of humans. Not surprisingly, the chromograms of robot users generally exhibited far more regularity than human users. Fig. 12 shows an example of a subset of a comment chromogram for one such program, "AFD_Bot". (AFD stands for "Articles for Deletion".) While the AFD_Bot is not human, we include this image because the bot was written by a human admin and thus may be considered part of the overall human editing strategy on Wikipedia, marking the extreme end of the systematic type of activity.

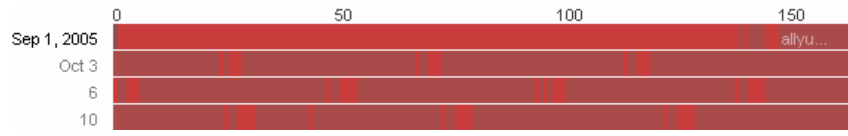

Fig. 12. Title chromogram for "AFD bot". Note the small range of colors and regular rhythm of edits.

\subsection{Reactive Activities}

A second category of activities seemed to be reactive. For these activites, which were essentially driven by external events and time-sensitive, users seemed to set themselves up to watch over certain aspects of the site. Reactive editing has already been described by [16] in the case of vandalism.

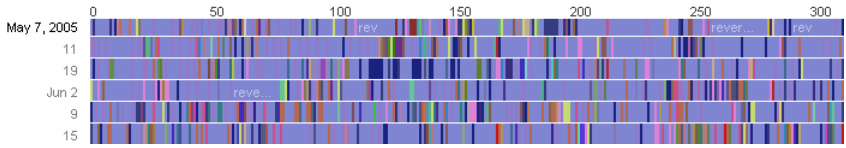

Fig. 13. Comment chromogram: Reverting vandalism and other bad edits

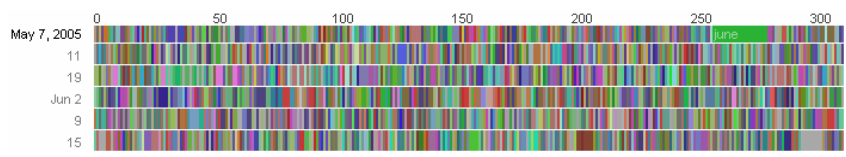

Fig. 14. Title chromogram for same edits in Fig. 13.

Fig. 13 shows a comment chromogram for a user whose focus is reversion. The diagram contains about 2,000 edits. The light blue corresponds to comments beginning with "revert." For a contrast, consider the corresponding title chromogram for 
the same series of edits, in Fig. 14. There is no clear pattern, since the reversions occur on a diverse set of pages. (It is interesting to compare these two diagrams with Figures 7 and 8, where the reverse is true: the titles were uniform and the comments diverse.) An analysis of all 509 admins showed that the behavior in Fig. 13 is extreme but not unique. For seven admins in our sample, the majority of edit comments referred to either reversion or vandalism ${ }^{2}$. For $152(30 \%)$ of admins, at least $10 \%$ of edit comments were revert- or vandalism-related.

As with systematic edits, we see task switching and a diverse set of edits apart from the core focus task. Although the majority of edits are marked as reverts, there is other activity interspersed as well. Articles are "wikified" with correct syntax and links, and some general content is added.

Vandalism is by no means the only type of event that drives edits. The arrival of a new user has obvious importance for the Wikipedia community, and we observed several editors who took on the task of welcoming new users. (This corroborates the engagement between experienced users and newcomers described in [4].) There are also many events that are related to steps in various Wikipedia. Requests for peer reviews, featured article status, admin privileges, the removal of admin privileges, and arbitration are all events that drive subsequent actions.

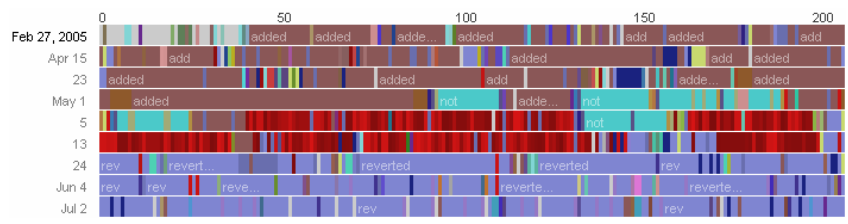

Fig. 15. Comment chromogram with about 2,000 edits between February and July 2005. Longterm task focus switches are evident. Brown: categorization; blue: reverts.

\subsection{Mixtures of Strategies}

For clarity, the preceding section focused on portions of edit histories that illustrated a single task focus. Many admins, however, seemed to switch between task foci at a large time scale. Fig. 15 shows a typical example. The brown, red, and cyan areas correspond to categorization activities. The blue area at bottom, on the other hand, represents vandalism repair.

\section{Discussion}

What do these editing patterns tell us about how admins allocate their effort? At a broad scale, we have seen that many of them make a vast number of edits. At the same time, we observe a high degree of diversity within a single editor's history, with sustained uninterrupted attention to a single article or task type being uncommon.

\footnotetext{
${ }^{2}$ We counted all comments that contained the text "revert" or "vandal," or began with "rv," a common abbreviation for reversions.
} 
On the other hand, given that the individuals in our sample sometimes made upwards of 10,000 edits, the diversity of edit types raises a question. How is it that the self-allocation of effort by individuals can be efficient, as described by Benkler [2]? Making a good decision a few times might be easy, but making 10,000 smart decisions seems hard by any standard. In other words, there seems to be an inherent tension between Benkler's hypothesis that large-scale peer-production systems should break work into fine-grained units, and the desire for workers to make as few decisions as possible about what to work on next.

A resolution to the mystery may lie in our second main observation: that editors tend to have focal tasks that, despite interruptions, take up a significant percentage of their time. In some cases they may make a single decision to react to a certain type of event, such as the arrival of a new user or an act of vandalism. In other cases, the editor may join a Wikiproject or find a list of pages that need work, make a single decision to work on every item in a list, and then systematically move through a set of tasks that others have aggregated.

In each case, the editor has greatly reduced their own cognitive burden. Instead of making a separate decision about the value of each individual potential edit, they make a decision to focus on a particular type of work and then perform a large set of related tasks. For reactive activity this set of related tasks may come through semiautomatic filtering mechanisms such as watchlists or posts to special pages such as "votes for deletion". In other cases, such as Wikiprojects, a purely human phenomenon is occurring: one set of people is collating, listing, and sorting tasks, which are then performed by others.

These cases form an interesting example of non-hierarchical flow of information about where work is needed. Viewed in this light, tasks such as categorization of pages are as much about organizing work as they are about organizing content. That is, the beneficiaries of Wikipedia's many lists and categories are not just readers looking to make connections between topics but also editors looking for new work items.

An important direction for future work would be to deepen our analysis via qualitative research methods. Although part of the impetus for our research was to look at quantitative issues, we believe our findings have raised questions that may be best answered through qualitative investigations. A second future direction would be to make comparative studies, for example between new and experienced users or active non-admins versus admins. Such comparisons might lend quantitative support (or could falsify) hypotheses about the trajectory from new user to admin.

\subsection{Chromograms}

Considering the simplicity of the color mapping, the chromogram technique seems to be surprisingly effective. One explanation is that even a random encoding technique will reveal some patterns, e.g., with high likelihood distinguishing between a run of 10 identical tokens and a sequence of 10 distinct tokens. Furthermore, coloring based only on the beginning of the word means that stemming occurs automatically, abbreviations are often handled correctly, and effects of alphabetical ordering are clear.

Some of the benefits of chromograms come from the consistency of the coloring across users. In our experience with the tool, we quickly grew to recognize colors associated with common words such as "list" and "revert." Moreover, we were able to 
recognize similarities across users: having seen one user who made many comments starting with "birth" and "death," it was easy for one of the authors to spot that pattern in other users' chromograms.

Another explanation may lie in the nature of Wikipedia titles and comments. Comments from administrators often employ conventional terminology ("revert", "fix", "wikify", etc.) and rarely start with content-free words such as "the" or "of". Many article titles start with a helpful keyword: for instance "list" for lists, or "HMS" for British ships. These conventions add structure to the data that helps make the visualization legible. The technique might therefore be less successful with less well-structured text.

Given the initial promise of the chromogram technique, it is natural to look for other applications and possible extensions. One direction for future research is simply to test the tool by applying it to other domains that feature timestamped snippets of text such as commits to software projects or email headers.

There are also several possible improvements to the basic technique. It would be interesting to explore the space of color mappings. What are the optimal constants for the letter-to-color translation (i.e., for the code in Appendix One)? What schemes are best for users with color deficiencies? More broadly, are there useful non-alphabetical schemes, for instance based on custom ontologies? (Our initial experiments in this direction using the Wikipedia category scheme gave mixed results.) There is also room for improvement in the simple layout. Would it be possible to show additional dimensions such as the size of an edit?

\subsubsection{Directions for Design}

One set of implications for design comes from the ways in which Wikipedia editors have reinvented wiki technology. Wikis are remarkably flexible, and so the community can use the same interface that supports articles to create, without reprogramming, de facto discussion boards (as in Talk pages) and bug databases (as in lists of needed work). Examining the conventions the community has created may provide design ideas for other systems where such features would need to be programmed.

In fact it is illuminating to compare the Wikiproject pages with standard bug databases in open source projects. Wikiprojects can easily describe a set of overlapping concerns, while providing a central page and discussion of that topic. While bug databases such as Bugzilla support overlapping concerns to some degree through tagging, they do not offer users the same sense of purpose and "place" that Wikiprojects do. Perhaps it would help to let users create special project areas with their own discussion sections and membership lists.

A second set of design implications stems from the systematic list-driven activites we observed. It might be useful, for example, to allow users to declare their intentions to work through a list. Given that the pervasive use of alphabetically ordered lists may actually be biasing which pages get edited, it might also be worthwhile to include different sorting mechanisms. For example, putting the oldest items at the top of a list might distribute effort more evenly.

At the same time, we may draw lessons from the flexiblility of wiki technology. The Wikipedia community has been able to coopt and reinvent [15] the basic wiki framework for many coordination tasks. When the underlying technology does change, it is often to formalize methods that have been "prototyped" by Wikipedia 
users. In this sense Wikipedia can be viewed as a grand experiment in participatory design [12]. Finding ways to add rapid user prototyping capabilities to other communities is an interesting general area for exploration.

\section{Conclusion}

This paper described a broad study of Wikipedia administrator activity. To explore edit histories of administrators, we introduced a new visualization technique, the chromogram. The new technique is a space-efficient way of displaying sequences of words and phrases by mapping text to color using an alphabetical code.

Despite the simplicity of the coding scheme, the chromogram technique turned out to be effective in distinguishing between various patterns of activity, and helpful in viewing small- and large-scale structures. Because of its simplicity and generality, the technique may be useful in contexts aside from Wikipedia.

Through examination of chromograms of editing patterns, we found that admins usually switched between multiple tasks, rarely concentrating on the same type of work continuously. At the same time, certain focal tasks seem to occupy a significant proportion of their time. These focal tasks fell into two types. Some were systematic; one tell-tale signature of such activities is a long alphabetically ordered sequence of article titles in the edit history. Other tasks were reactive, as admins reacted to vandalism or requests from other Wikipedia users.

While other studies have examined reactive activities such as vandalism, the existence of systematic, sustained sequences of edits has received less attention. The existence of such organized behavior suggests that devices such as lists, categorization schemes, and Wikiprojects play a strong role in what individual admins choose to work on. The fact that these organizational devices exist helps resolve a tension in peerproduction work: having many fine-grained tasks has benefits, but to reduce the cognitive burden on workers, it is important to provide organizing devices that help them allocate their time at a higher level of granularity than an individual task.

\section{References}

1. Begole, J., Tang, J., Smith, R., Yankelovich, N.: Work Rhythms: Analyzing Visualizations of Awareness Histories of Distributed Groups. In: CSCW 2002 (2002)

2. Benkler, Y.: Coase's Penguin, or, Linux and The Nature of the Firm. 112 Yale Law Journal 369 (2002)

3. Bosch, R., Stolte, C., Rosenblum, M., Hanrahan, P.: Performance Analysis and Visualization of Parallel Systems using SimOS and Rivet: A Case Study. In: Int'l Symposium on High Performance Computer Architecture (2000)

4. Bryant, S., Forte, A., Bruckman, A.: Becoming Wikipedian: Transformation of Participation in a Collaborative Online Encyclopedia. In: GROUP 2005 (2005)

5. Butler, B., Sproull, L., Kiesler, S., Kraut R.: Community Effort in Online Groups: Who Does the Work and Why? In: Weisband, S., Atwater, L. (eds.) Leadership at a Distance (2002)

6. Forte, A., Bruckman, A.: Why do people write for Wikipedia? Incentives to contribute to open-content collaboration. In: Proceedings of GROUP 2005 workshop (2005) 
7. GNU Free Documentation License v. 1.2 (2002), Downloaded from www.gnu.org/ copyleft/fdl.html

8. Keim, D.: Pixel-Oriented Database Visualizations. SIGMOD Record 25(4) (1996)

9. Lakhani, K., Wolf, R.: Why Hackers Do What They Do: Understanding Motivation and Effort in Free/Open Source Software Projects. In: Perspectives on Free and Open Source Software, MIT Press, Cambridge (2005)

10. Lave, J., Wenger, E.: Situated Learning: Legitimate Peripheral Participation. Cambridge University Press, Cambridge (1991)

11. Mockus, A., Fielding, R., Herbsleb, J.: Two Case Studies of Open Source Software Development: Apache and Mozilla. ACM Trans. Software Engineering and Methodology 11(3), 309-346 (2002)

12. Schuler, D., Aki, N. (eds.): Participatory Design: Principles and Practices. LEA (1993)

13. Seeberger, M., Kuhn, A., Girba, T., Ducasse, S.: Chronia: Visualizing how developers change software systems. In: CSMR 2006 (2006)

14. Sherif, M., Harvey, O.J., White, J., Hood, W., Sherif, C.W.: Intergroup conflict and cooperation: the robbers cave experiment. University Book Exchange, Norman, OK (1961)

15. Sproull, L., Kiesler, S.: Connections: new ways of working in the networked organization. MIT Press, Cambridge (1991)

16. Viégas, F., Wattenberg, M., Dave, K.: Studying Cooperation and Conflict between Authors with history flow Visualizations. In: CHI 2004 (2004)

17. Viégas, F., Wattenberg, M., Kriss, J., van Ham, F.: Talk Before You Type: Coordination in Wikipedia. In: HICSS 40 (2007)

18. Wagner, C.: Breaking the Knowledge Acquisition Bottleneck Through Conversational Knowledge Management. Information Resources Management Journal 19(1), 70-93 (2006)

19. Ware, C.: Information Visualization: Perception for Design. Academic Press, London (2000)

20. Wattenberg, M.: Arc Diagrams: Visualizing Structure in Strings. In: IEEE InfoVis 2002 (2002)

21. Wikipedia: Active Administrators, English (September 2006), http://en.wikipedia.org/wiki/ Wikipedia:List_of_administrators

22. http://en.wikipedia.org/wiki/Wikipedia:List_of_administrators_by_edit_count

23. http://en.wikipedia.org/wiki/Special:Statistics 